\title{
STRONG DOUBLING CONDITIONS
}

\author{
STEPHEN M. BUCKLEY
}

Abstract. We show that the class of strong doubling measures depends essentially on the parameter $t$, and that the measure of the boundary layer of a QHBC domain decays geometrically, if the measure is suitably strong doubling.

Mathematics subject classification (1991): 28A75, 42B25.

Key words and phrases: Doubling condition, QHBC domain, maximal functions, covering lemmas.

\section{REFERENCES}

[Bo] B. BoJARski, Remarks on Sobolev imbedding inequalities, Proc. of the conference on Complex Analysis, Joensuu 1987, Lecture Notes in Math. 1351, Springer-Verlag, Berlin, 1989, pp. 52-68.

[BKL] S.M. Buckley, P. Koskela, And G. Lu, Subelliptic Poincaré inequalities: the case $p<1$, Publ. Mat. 39 (1995), 313-334.

[BO] S.M. BuCKLEY AND J. O'SHEA, Weighted Trudinger type inequalities, preprint.

[GO] F.W. GEHRING AND B. OSGOOD, Uniform domains and the quasihyperbolic metric, J. Analyse Math. 36 (1979), 50-74.

[G] M. DE GuZMÁN, Differentiation of integrals in $\mathbb{R}^{n}$, Lecture Notes in Math. 481, Springer-Verlag, Berlin, 1975

[HKM] J. HEINONEN, T. KILPELÄINEN, AND O. MARTIO, Nonlinear potential theory of degenerate elliptic equations, Oxford Univ. Press, Oxford, 1993.

[JM] P.W. Jones AND N.G. MAKarov, Density properties of harmonic measure, Ann. of Math. (2) 142 (1995), 427-455.

[KR] P. KosKela AND S. RoHDE, Hausdorff dimension and mean porosity, Math. Ann. 309 (1997), 593-609.

[MV] O. Martio AND M. Vuorinen, Whitney cubes, p-capacity, and Minkowski content, Expo. Math. 5 (1987), 17-40.

[M] A.P. MoRSE, Perfect blankets, Trans. Amer. Math. Soc. 6 (1947), 418-442.

[SS] W. Smith AND D.A. STEGENGA, Exponential integrability of the quasihyperbolic metric in Hölder domains, Ann. Acad. Sci. Fenn. Ser. A I. Math. 16 (1991), 345-360.

[S1] E.M. STEIN, Singular Integrals and Differentiability Properties of Functions, Princeton University Press, Princeton, 1970.

[S2] E.M. StEIN, Harmonic Analysis: real-variable methods, orthogonality, and oscillatory integrals, Princeton Univ. Press, Princeton, 1993. 